\title{
Design and Control of a Compliant Stage with Reconfigurable Dynamic Characteristics by Incorporated Rubber Shear Damper
}

\author{
Kung-Han Lee ${ }^{\mathrm{a} 1}$, Kuo-Shen Chen ${ }^{\mathrm{b} 1}$, and Yun-Hui Liu ${ }^{\mathrm{b} 2}$ \\ ${ }^{\mathrm{a}}$ Graduate Student, ${ }^{\mathrm{b}}$ Professor, \\ ${ }^{1}$ Department of Mechanical Engineering, National Cheng-Kung University, \\ No. 1, Daxue Rd., East Dist., Tainan City 701, Taiwan (R.O.C.) \\ ${ }^{2}$ Department of Mechanical Engineering, Southern Taiwan University of Science and Technology, \\ No. 1, Nantai St., Yungkang Dist., Tainan 71005, Taiwan (R.O.C.) \\ *Corresponding Author: N16074352@gs.ncku.edu.tw
}

\begin{abstract}
This paper presents the dynamic analysis and control system design of a compliant stage with reconfigurable dynamic characteristics, consist of a notch-based structure and a mechanical amplifier. Generally, compliant stages are usually fabricated of metallic structures. Such an approach could yield excellent performance in many applications with simple feedback controls incorporated. However, the system dynamic cannot be changed once the geometry of the stages is designed. In this work, we propose a dynamic characteristics reconfigured approach by incorporating polydimethylsiloxane (PDMS) layer to modify the dynamic characteristics of compliant structures. In order to implement the approach, a compliant stage is designed and realized. Essential performance and dynamic tests are conducted to examine the functional performances and dynamic characteristics of both the original and PDMS-modified stages. The dynamic testing results indicate that the PDMS materials can significantly increase the stiffness and damping coefficient of the stage for $45 \%$ and $6.7 \%$, respectively. Finally, for assessing the possible advantage in precision control by using this structural reconfiguration approach, controller systems are designed based on loop transmission shaping and positive acceleration, velocity and position feedback methods. The experimental results demonstrate that settling time is indeed improved from $55.3 \mathrm{~ms}$ to $36.2 \mathrm{~ms}$ after the dynamic characteristics are adjusted.
\end{abstract}

Keywords: Compliant stage, PDMS rubbers, Reconfigurable dynamics, Positioning control.

\section{Introduction}

Compliant stages are critical subsystems in precision motion control for various industrial applications ranged from machining tool handling to sub-micron metrology ${ }^{(1-2)}$. Generally, they are fabricated by using conventionally machining methods such as wire cutting of metallic structures. Such an approach has advantages of high stiffness and high reliability and this could yield excellent performance in many applications with feedback controls incorporated. However, the dynamic characteristics is usually unalterable once the geometry of the stages is designed. For example, for increasing stiffness of compliant structure $200 \%$, the structural length should be redesigned and decrease to $70 \%$ of the original one if the other parameters of geometry were fixed ${ }^{(3)}$. Note, the low adjustable capability of dynamic characteristics leads to a major challenge of operating stages in different applications. As far as the cost of stage fabricated is concerned, a low adjustable capability of dynamic characteristics provides only certain application and results in an increase in actual application costs. In this sense, the stage design with reconfigurable dynamic characteristics could be easier to achieve verities application requirement.

For past decades, many techniques have been proposed to reconfigure the dynamic characteristics of compliant stages ${ }^{(4-5)}$. However, it is challenging to add traditional dampers due to the spatial consideration in many small-scale ultra-precision applications. As a result, how to effectively enhance adjustable capability of the dynamic 
characteristics of compliant stage under space constraint is a non-trivial issue in precision positioning control. Polydimethylsiloxane (PDMS), due to its low stiffness and high damping nature, can be incorporated into compliant structures to reconfigure the system dynamics ${ }^{(4)}$. It is worthwhile to mention that PDMS is generally applied as biomedical materials ${ }^{(6)}$ and has new interests as precision positioning application ${ }^{(7)}$, recently. Previously, $\mathrm{Yu}^{(4)}$ successfully verified the damping enhancement mechanism through 3D printing structure. It should be noted, PDMS has stiffness and damping which is similarly with the 3D printing materials. In this sense, PDMS would also affect the system stiffness of the 3D printing structure significantly.

Motivating by the literature mentioned above, a dynamic characteristics reconfigured approach of metallic structure is proposed by incorporating PDMS in this work. The overall research flow is shown is Fig. 1. The developed of this stage with revisable dynamic characteristics can be implement with three steps. First, essential performance and dynamic tests are conducted to examine the functional performance and dynamic characteristics of the stage containing the original one and the one with PDMS incorporated. Second, for verifying the proposed the dynamic characteristics reconfigured approach, a feedback controller is designed based on loop transmission shaping to conduct a series of position experiments. Finally, for obtaining a better performance of control system, positive acceleration, velocity and position feedback controller is designed and applied in this work.

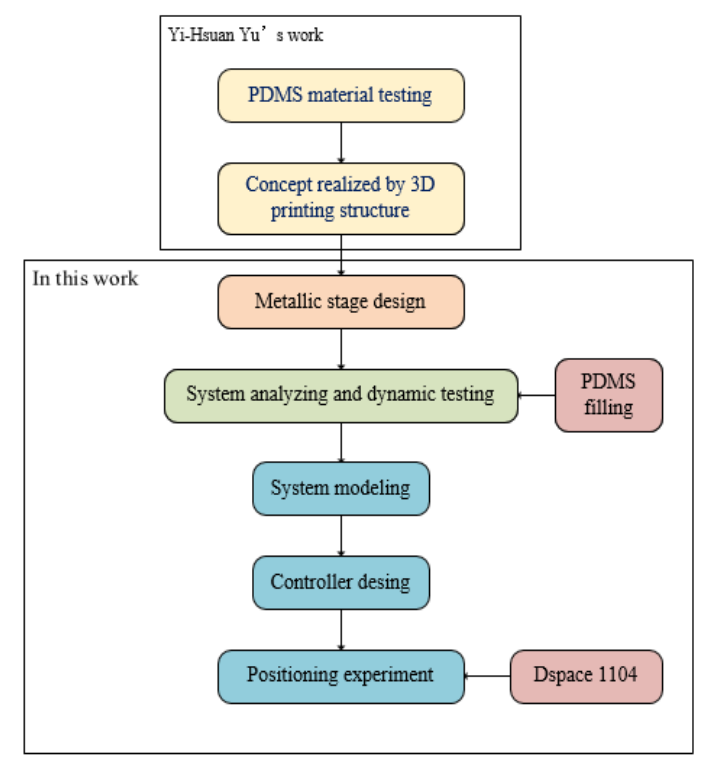

Fig. 1. Overall research flow
The rest of this paper presents the testing, and control of the stage in detail. A brief description on the stage design, realization and PDMS filling is addressed in chapter II and followed by the preliminary dynamic characterization results and presented the preliminary dynamic behavior of stage integrated with rubber is demonstrated in Section III. Subsequently, feedback controllers are design in Section IV and Section V briefly discusses the experiment results. Finally, Section VI present the conclusion of this work and provides future perspective and concludes this work.

\section{Approach}

\subsection{Stage design and realization}

In this section, the well-defined compliant stage ${ }^{(8)}$ and the mechanical amplifier are brief presented for evaluating the proposed adjustable capability enhancement strategy. Here, the design of which the XY fully decoupled parallel stage shown in Fig. 2 consists of four individual limbs connected at the center point in parallel. Each limb provides essential compliance and serves as a displacement amplification mechanism. The final dimensions are listed in Table 1 and the schematic plot of the structure is shown in Fig. 3. $\mathrm{Li}^{(3)}$ et al. has already conducted numerical analysis about this displacement amplification for a complete compliance analysis based on the potential energy analysis and Castigliano's theorem, the derivation of the stiffness. The technical detail on design analysis can be found elsewhere $e^{(3-4,8)}$. A finite element analysis performed by Abaqus is conducted in this work for accessing the structural compliance and to evaluate the state of stress during operation. The material used for constructing the stage is the conventional Aluminum 5052 with a Young's modulus of 69.3GPa and a Poisson's ratio of 0.33. As shown in Fig. 4, the structure at notch hinges have the highest stress level as expected and the first three natural frequencies are 94.2, 130.36, and 151.24Hz. Since the second resonant frequency is much higher than the first, the stage can be treated as the equivalent of a single degree of freedom system during operation without loss of generality. The amplification ratio and input-output stiffness of structure are all obtain by finite element analysis, as listed in Table 2. The error may result from the non-ideal boundary. Although PDMS should be added in the portions where the maximum stress is observed for efficiently 
dissipating energy to change dynamic characteristics of the stage, the PDMS is incorporated in the position shown in Fig. 5. in the work instead due to the spatial concern. During movement, this design will still generate a shear force on PDMS and the dynamic characteristics changing effect should be realized as expected.

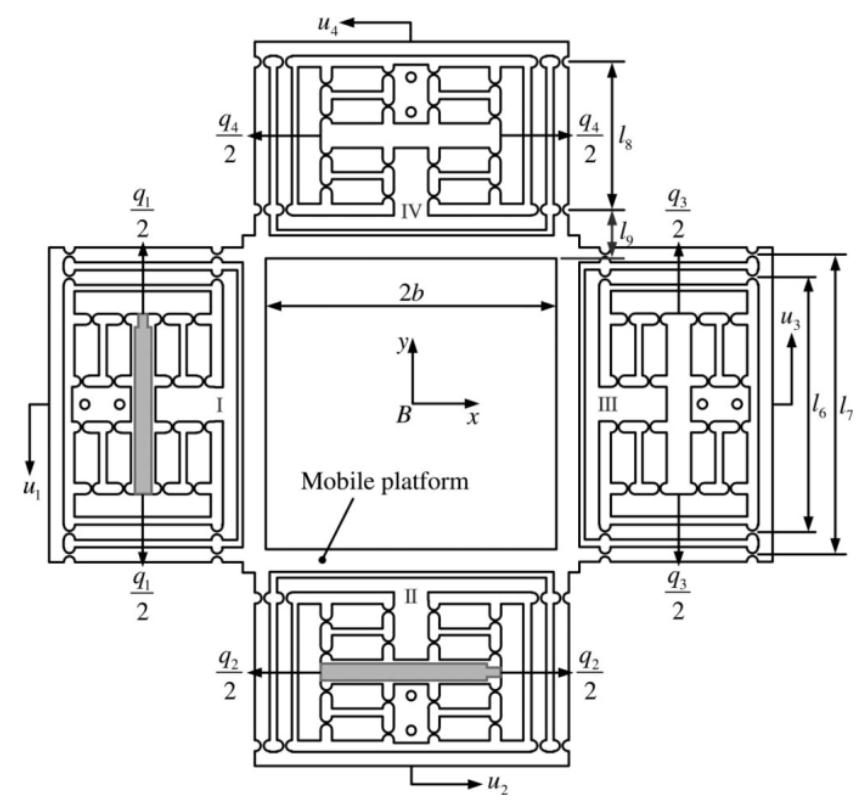

Fig. 2. XY fully decoupled parallel stage ${ }^{(8)}$

Table 1. dimensions of the stage (mm)

\begin{tabular}{|c|c|c|c|c|c|c|c|}
\hline$r$ & $t$ & $l_{x}$ & $l_{y}$ & $w$ & $h_{1}$ & $l_{1}$ & $l_{2}$ \\
\hline 8 & 1.2 & 60 & 5 & 10 & 5 & 14 & 37.4 \\
\hline
\end{tabular}

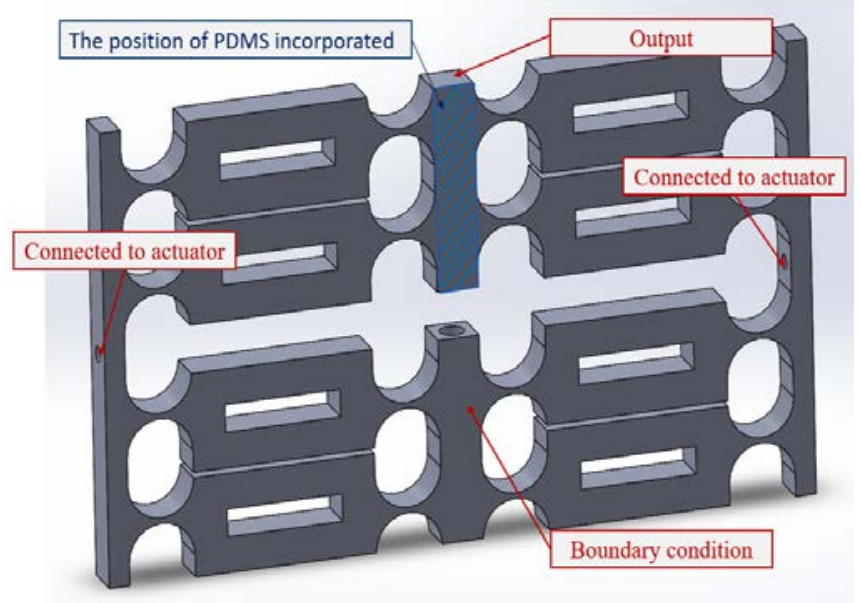

Fig. 3. The schematic plot of the compliant structure

\section{$2.2 \quad$ Experimental setup}

A brief functional block diagram of the stage control is shown in Fig 6(a) and the entire experimental setup is

shown in Fig 6(b) containing two AVM40-20 voice coil motors of which maximum output force is $54 \mathrm{~N}$ as the actuators, two ASP-10-CTR capacitive probes of which measurement range is $254 \mu \mathrm{m}$, resolution is $10 \mathrm{~nm}$ and bandwidth is $10 \mathrm{kHz}$ used for characterizing the input and output displacements, a compliant stage and a dSPACE controller with 14-bit $\mathrm{A} / \mathrm{D}$ and 14-bit $\mathrm{D} / \mathrm{A}$ for data acquisition and for sending out controlled output for controlling the motion and the experiments are all executed with sampling rate of $10 \mathrm{kHz}$. The voice coil motors actuate the stage and the capacitive probes for sensing the input and output movement. Therefore, major performances such as input-output stiffness, amplification ratio, natural frequencies, and even damping ratio, can be obtained. Moreover, real time control system experiments are implemented by dSPACE FPGA controller. This test system is firstly used to obtain the system dynamics and then, for performing the evaluation on stage control for validating the proposed conceptual design.

(a)

Mode 2: $f_{2}=130.36 \mathrm{~Hz}$

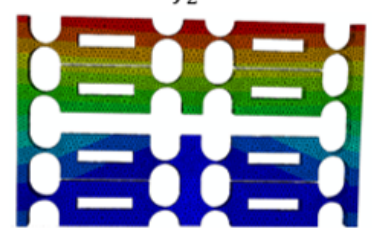

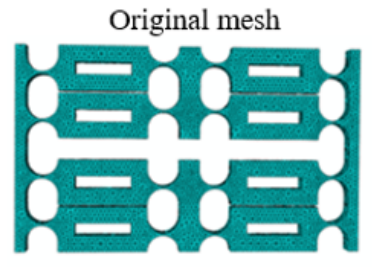

Mode 1: $f_{1}=94.2 \mathrm{~Hz}$

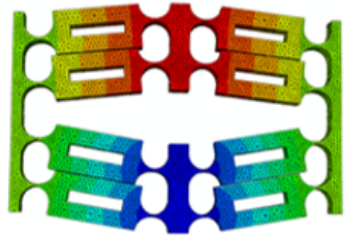

Mode 3: $f_{3}=151.24 \mathrm{~Hz}$

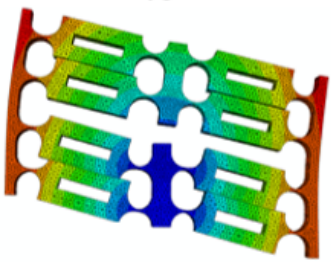

(b)

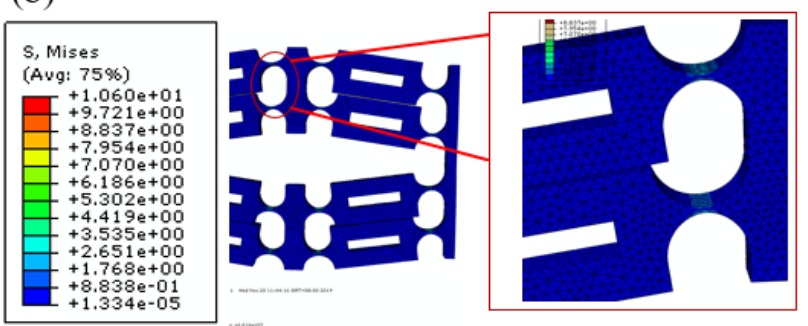

Fig. 4. The finite element simulation of the stage. (a)The original mesh and the mode shapes of the first three modes and (b) The von Mises stress contour.

Table 2. The amplification ratio and input-output stiffness

\begin{tabular}{|c|c|c|}
\hline & Amplification ratio & input-output stiffness \\
\hline $\begin{array}{c}\text { Finite element } \\
\text { analysis }\end{array}$ & 13.91 & $0.3829(\mu \mathrm{m} / \mathrm{N})$ \\
\hline
\end{tabular}



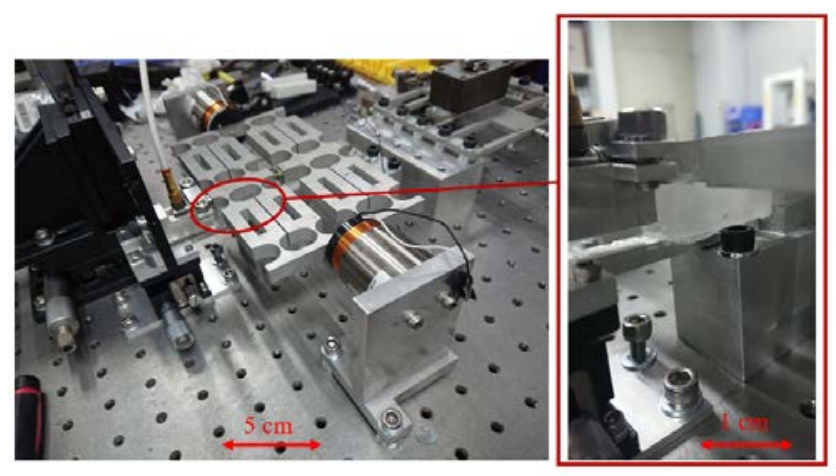

Fig 5 the position of PDMs incorporated

(a)

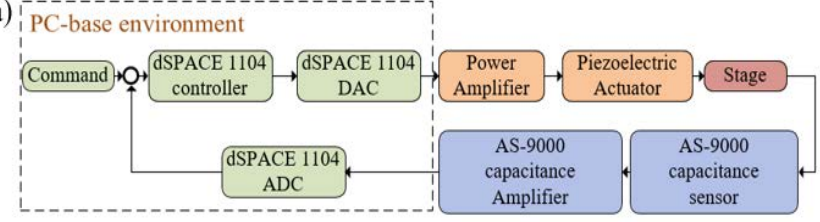

(b)

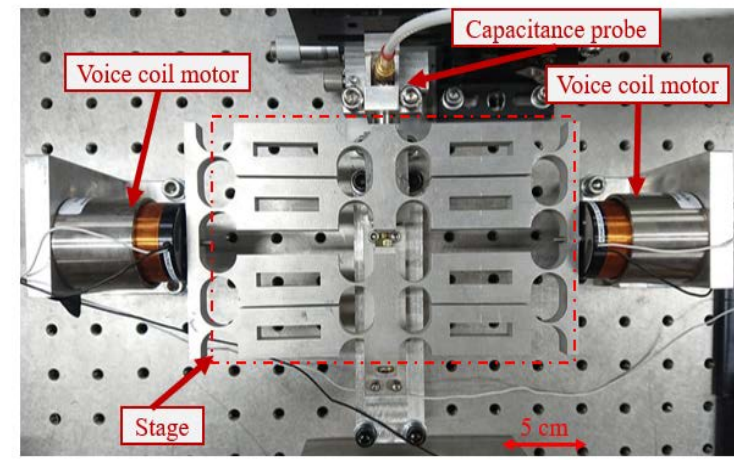

Fig. 6. (a) Experimental setup and (b) Block diagram of the stage control

\section{System dynamic and modeling}

\subsection{Original stage dynamics}

The stage is modeled as a single degree of freedom vibration system with a mass, $\mathrm{M}$, a damper with damping coefficient, C, and spring, $\mathrm{K}$, By force equilibrium, it can be shown that the transfer function, $G_{\text {stage }}(\mathrm{s})$, of this stiffness element can be expressed as

$$
G_{\text {stage }}=\frac{1}{M s^{2}+C s+K}
$$

The mass $\mathrm{M}$ in the model is taken as the mass of the aluminum stage, $446 \mathrm{~g}$. The stiffness is $0.34 \mu \mathrm{m} / \mathrm{N}$ which is determined by examining the open-loop step response and is closed to the one obtained by finite element analysis. The damping coefficient $\mathrm{C}$ is hard to be obtained analytically and is finally experimentally determined by frequency sweeping. Experimental results and simulations are shown in Fig. 7 and consider the actuator system as one order system which has the expression of

$$
G=G_{a c t}=K_{a}=\times \frac{2 \pi \times 200}{s+2 \pi \times 200}
$$

which $K_{a}$ is the coefficient of amplifier. The transfer function of the entire plant can be finally expressed as

$$
G_{\text {plant }}=\frac{a}{s^{3}+b s^{2}+c s+d}
$$

which $\mathrm{a}=5.06 \times 10^{8}, \mathrm{~b}=1278, \mathrm{c}=3.121 \times 10^{5}$ and $\mathrm{d}=$ $3.584 \times 10^{8}$

(a)

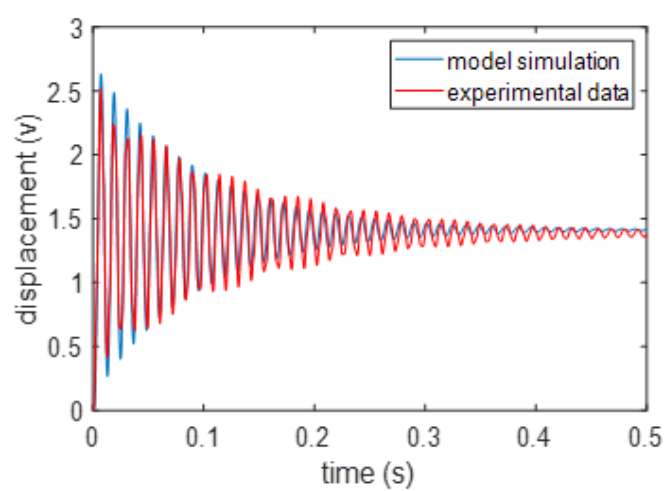

(b)
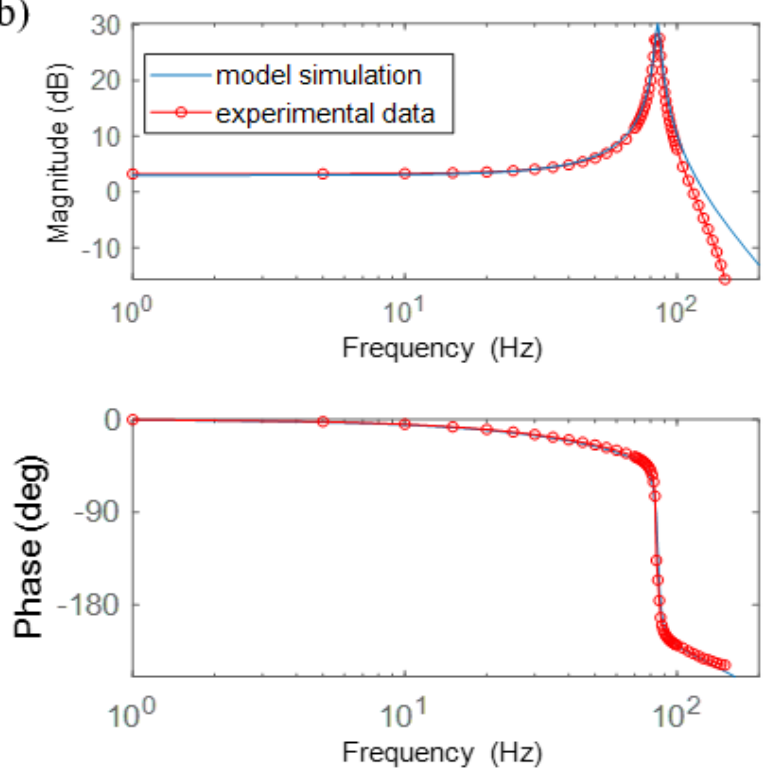

Fig. 7. (a) Open-loop step response and (b) frequency sweeping 


\subsection{Dynamic characterization of modified stage}

It is also important to perform dynamic characterization of the stage with PDMS incorporated. The stiffness and damping are obtained by the same method as of original stage. Here, a series of step response experiments are conducted to test the variety of the system dynamic to valid the concept that the dynamic characteristics is adjusted after PDMS incorporated with different preload pressure. From the results of step response test, the damping coefficients are obviously changed as shown in Fig. 8. Moreover, the stiffness of the system can be increased $280 \%$ from the original one. Nevertheless, the damping ratios aren't enhanced as well due to the increasing of stiffness. Although, there can be several models which altered from the original one, we first present one kind of dynamic characteristics reconfigured stage in this work. The corresponding preload pressure of PDMS is chosen with trial and error method. For a comparative study, the frequency sweeping experimental results of this adjusted model and the original one are shown in Fig. 9.

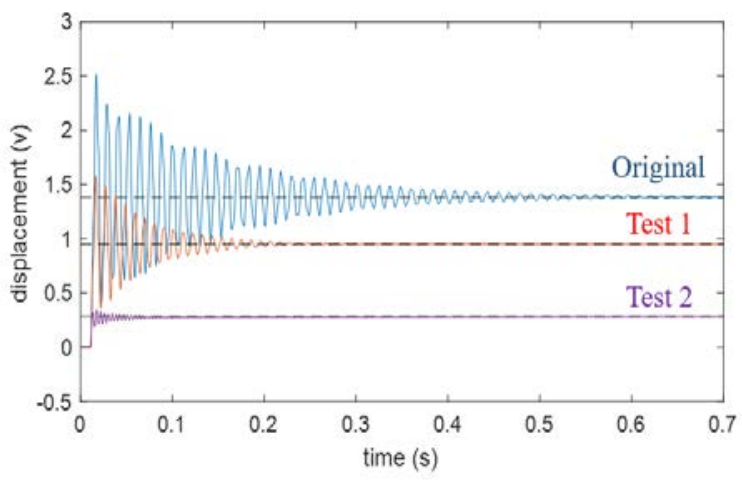

Fig. 8. Step response corresponding to different preload pressure of PMDS
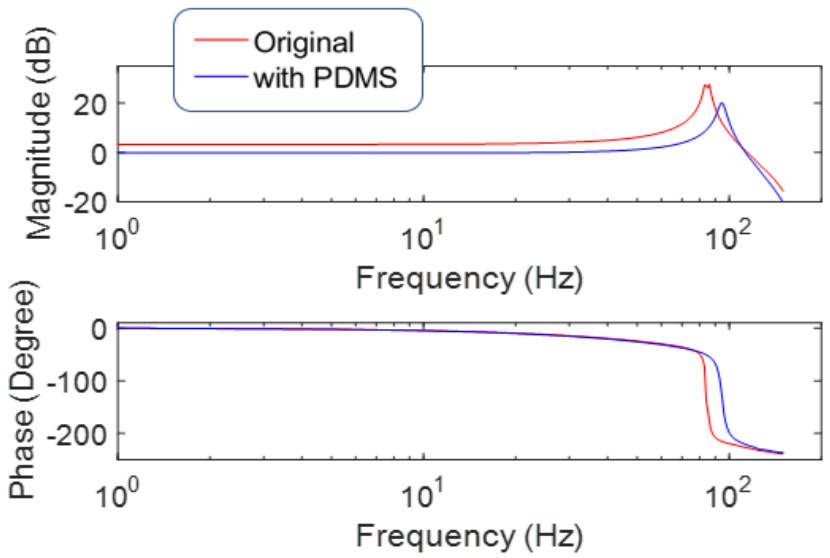

Fig. 9. Frequency sweeping experiment of original model and dynamic characteristics reconfigured model

\section{Control system design}

In order to verify this approach, three feedback controllers are design and adopted. In this work, we first present typical PID controller. Second, a loop transmission shaping (L.T.) method ${ }^{(9)}$ which designed by shaping the loop transmission of the system in frequency domain is used to achieve better performance of close loop system. After shaping loop transmission into desired from, the performance of the close loop system can be predicted. Finally, the positive acceleration, velocity and position feedback (PAVPF) controller ${ }^{(5)}$ is applied to the systems to achieve better performance. The block diagram of the system with the loop transmission shaping is shown in Fig. 10. The loop transmission is defined as

$$
T_{\text {open }}=C_{l t}(s) G_{\text {plant }}
$$

where $\mathrm{G}$ and $\mathrm{C}$ are the transfer function of the system and the controller containing notch filter to reducing the lightly damped resonant vibration and PI controller to improve the control bandwidth. Therefore, the loop transmission controller has the expression of

$$
C_{l t}(s)=K_{p}\left(1+\frac{K_{i}}{s}\right)\left(\frac{s^{2}+\frac{d}{c} \omega_{n o t c h} s+\omega_{\text {notch }}^{2}}{s^{2}+\frac{1}{c} \omega_{\text {notch }} s+\omega_{\text {notch }}^{2}}\right)
$$

where $K_{p} \cdot K_{i} \cdot d \cdot c$ and $\omega_{\text {notch }}$ are adjustable parameters. For a comparative study and obtaining better performance of control system, the positive acceleration, velocity and position feedback (PAVPF) controller is incorporated to deal the low damping behavior. The block diagram of this concept is shown in Fig. 10 and the transfer function of the PAVPF controller can be expressed as

$$
C_{d a m p}(s)=\frac{\Gamma_{2} s^{2}+\Gamma_{1} s+\Gamma_{0}}{s^{2}+2 \xi \omega_{p} s+\omega_{p}^{2}}
$$

The technical detail on design analysis can be found elsewhere $^{(6)}$.

(a)

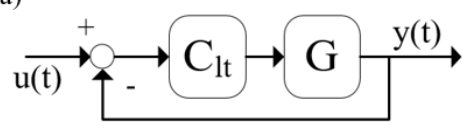

(b)

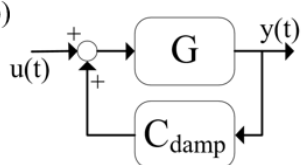

Fig. 10. (a) The block diagram of L.T. control and (b) The block diagram of PAVPF control 


\section{Experiment results}

\subsection{Step responses}

In this section, positioning experiments are conducted through the experimental setup as shown in Fig. 6. First, a series of typical step response experiments with PID controller for a $25.4 \mu \mathrm{m}$ step command of the system with PDMS incorporated are conducted and discussed. The control parameters of PID controller are tuned by trial and error method as Kp ranged from 0.003 to $0.011, \mathrm{Ti}=2.53 \times$ $10^{-4}$ and $\mathrm{Td}=6.325 \times 10^{-5}$. The experimental results are shown in Fig 11. It's obviously that the controller performance with only PID isn't good enough for high bandwidth required situation although the responses have nearly low overshoot. Moreover, the system becomes unstable after increasing a little of the proportional gain of PID controller. Second, loop transmission shaping controller experiments are conducted to determine the parameters of controller for all model containing the original system and the systems after dynamic characteristics modified by PDMS. Finally, the experiments of PAVPF controller are conducted. The experiments of PAVPF controller begin with step response and frequency sweeping. Experiment results containing with the open loop systems, the system with the PDMS and the one with both PAVPF and PDMS are shown in Fig. 12. It is apparently that the damping ratio is enhanced. Meanwhile, the output stiffness is increased from 0.001 to 0101 . It is worth noting that a L.T. controller is incorporated to deal with the tracking errors here. The detail of PAVPF controller parameters are list in Table 3 and the detail of the corresponding L.T. controller parameters are list in table 4. The experimental results of all step responses are shown in Fig. 13. For comparison study, all step responses are controlled to similar overshoot and rise time. Thus, we can distinctly identify how dynamic characteristics reconfigured affect the settling time. From experiment results, it is visibly that the systems with PDMS and PAVPF controller are less oscillated and the corresponding settling time are slightly shorter when they reach the destination. Moreover, compared with the PID controller, L.T. controllers have much shorter rise time. The detail of control system performance index of L.T. controller is listed in Table 4.

Table 3. The detail of PAVPF controller parameters

\begin{tabular}{|c|c|c|c|c|}
\hline$\Gamma_{0}$ & $\Gamma_{1}$ & $\Gamma_{2}$ & $\omega_{p}$ & $\xi$ \\
\hline 8338 & 43.36 & 0.18 & 171.8 & 0.34 \\
\hline
\end{tabular}

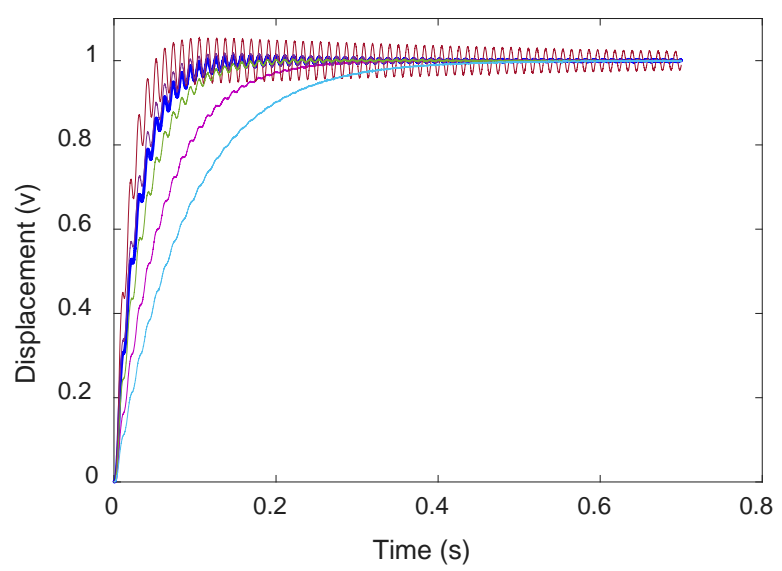

Fig. 11. Typical step response with PID controller

Table 4. Parameters of L.T. controller

\begin{tabular}{|c|c|c|c|c|c|}
\hline & $K_{p}$ & $K_{i}$ & $d$ & $c$ & $\omega_{\text {notch }}$ \\
\hline Original system & 0.047 & 1100 & 0.007 & 0.3 & 534.07 \\
\hline System with PDMS & 0.065 & 1470 & 0.03 & 0.3 & 609.47 \\
\hline $\begin{array}{c}\text { System with PDMS } \\
\text { and PAVPF }\end{array}$ & 0.051 & 1300 & 0.1 & 0.55 & 578.05 \\
\hline
\end{tabular}

(a)

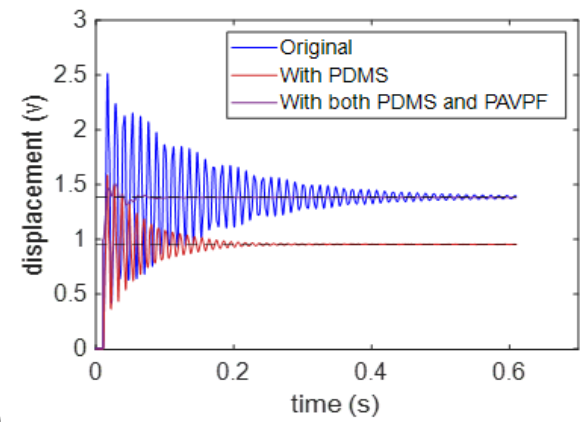

(b)
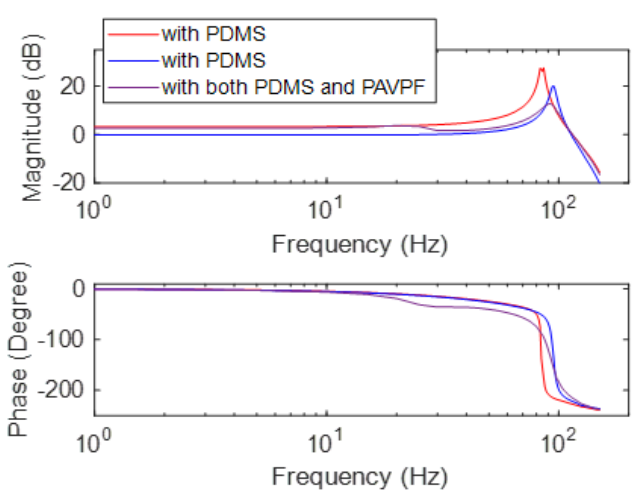

Fig. 12. (a) Open-loop step responses of all systems and (b) Frequency sweeping results of all systems 


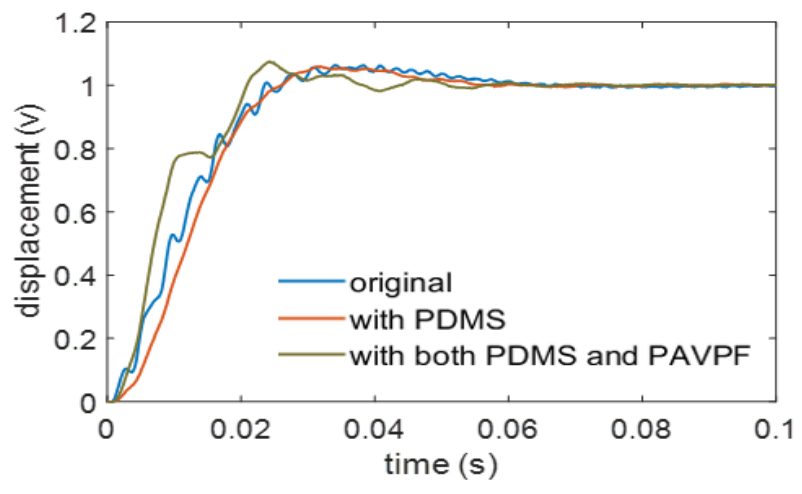

Fig. 13. Step response experimental results

Table 5. Control system performance index

\begin{tabular}{|c|c|c|c|}
\hline & Overshoot & Rise Time & Settling time \\
\hline Original system & $6.4 \%$ & $17.3 \mathrm{~ms}$ & $55.3 \mathrm{~ms}$ \\
\hline $\begin{array}{c}\text { System with } \\
\text { PDMS }\end{array}$ & $6 \%$ & $15.3 \mathrm{~ms}$ & $49.7 \mathrm{~ms}$ \\
\hline $\begin{array}{c}\text { System with } \\
\text { PDMS and PAVPF }\end{array}$ & $7.5 \%$ & $16 \mathrm{~ms}$ & $36.2 \mathrm{~ms}$ \\
\hline
\end{tabular}

\subsection{Sinusoid tracking experiments}

After determined the parameters of the loop transmission shaping controllers, real-time sinusoid trajectories tracking experiments are conducted. Fig. 14 and Fig. 15 demonstrates the comparison of the sinusoid tracking performance within different system under 1 and $50 \mathrm{~Hz}$, respectively. As Fig. 14 and Fig. 15 shown, it is apparently that the tracking error of the control system with the PDMS incorporated and the PAVPF controller is lower than the others. In this sense, the bandwidth of the control system with the PDMS incorporated and the PAVPF controller is higher than the others.

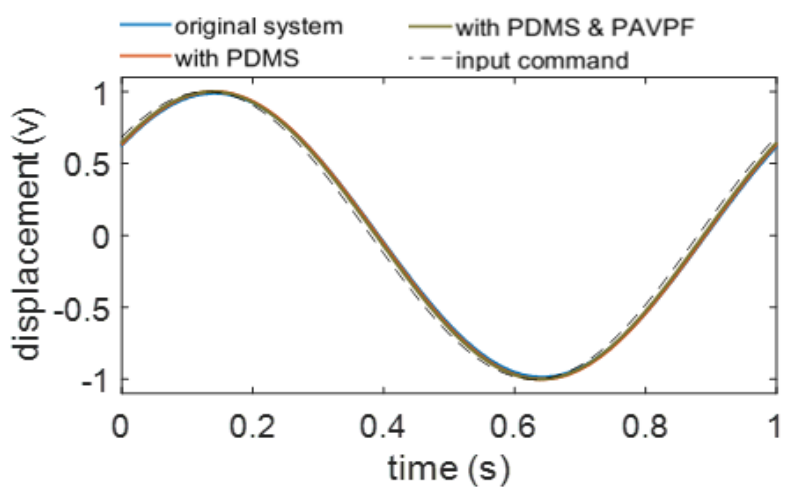

Fig. 14. Sinusoid tracking experiments under $1 \mathrm{~Hz}$

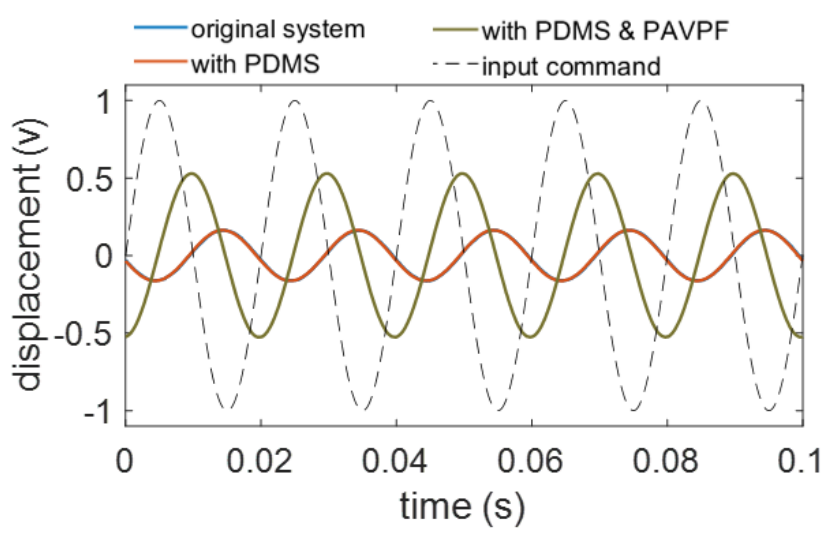

Fig. 15. Sinusoid tracking experiments under $50 \mathrm{~Hz}$

\section{Conclusion}

In this work, a compliant stage with alterable dynamic characteristics is proposed aiming for adjusting the system dynamics of compliant mechanism-based positioning stage for different applicated situations. By utilizing high material damping capacity of elastomers, PDMS, the stiffness is increased $45 \%$ without any geometry redesigned. Besides, after introducing PAVPF damping control system, it is possible to enhance the equivalent damping factor of the system. The process mentioned above leads to a better situation for controller design for achieving multiple application situation and better positioning performance. This study composed of a compliant amplifier design, the location of PDMS material researching and control system evaluating for verifying concept in adjusting the dynamic of the stage. The preliminary results indicate that the proposed method could effectively adjusting the dynamic characteristics of the stage. A two degree of freedom version design is underway for allowing a more practical evaluation. Meanwhile, it is suggested that the dynamic characteristics reconfigured concept can be integrated with CCD camera for certain high valued applications.

\section{Acknowledgment}

This work is currently supported by the Ministry of Science and Technology (MOST) of Taiwan under contact numbers MOST 105-2221-E-006-100-MY3, MOST 108-2221-E-006-209, MOST 108-2622-8-006-014 and MOST 108-2221-E-218-027. Contributions from Yi-Hsuan Yu, and Yu-Ching Li of National Cheng-Kung University (NCKU) during the early stages of this work are greatly appreciated. 


\section{References}

(1) J. W. Ryu, D.-G. Gweon, and K. S. Moon, "Optimal design of a flexure hinge based XY $\varphi$ wafer stage," Precis. Eng., vol. 21, no. 97, pp. 18-28, 1997.

(2) W. C. Wang, J. W. Lee, K-S Chen, and W-H Liu, "Design and vibration control of a notch-based compliant stage for display panel inspection applications,” Journal of Sound and Vibration, Vol. 333, pp. 2701-2718, May, 2014.

(3) Y. Li and Q. Xu, "A Novel Piezoactuated XY Stage With Parallel, Decoupled, and Stacked Flexure Structure for Micro-/Nanopositioning,” IEEE Transactions on Industrial Electronics, Vol. 58, no. 8, pp. 3601-3615, 2011.

(4) Y-H Yu, Design and Realization of a Polymer Compliant Stage with $3 D$ Printing and Rubber Damping, Master Thesis, National Cheng-Kung University, Taiwan, 2016

(5) $\mathrm{L}-\mathrm{L} \mathrm{Li}$, et al, "Positive acceleration, velocity and position feedback based damping control approach for piezo-actuated nanopositioning stages," Mechatronics, Vol.47, pp. 97-104, 2017

(6) Y-M Liao, Mechanical Properties Characterization of Soft Materials for Semiconductor Processing Equipments, Biomedical Measurements and Roll to Roll Transportations, Master Thesis, National Cheng-Kung University, Taiwan, 2008

(7) Lin, Pei-Chun, Yen-Feng Cheng, and Kuo-Shen Chen, "Design and Realization of a Novel Elastomer Characterization System for Precision Positioning Application," 2018 18th International Conference on Mechatronics-Mechatronika (ME), IEEE, pp. 1-6, 2018

(8) Y. Li and Q. Xu, "Design and Analysis of a Totally Decoupled Flexure-Based XY Parallel Micromanipulator," IEEE Transactions on Robotics, Vol. 25, no. 3, pp. 645-657, 2009

(9) D. F. Thompson and O. D. I. Nwokah, "Analytic Loop Shaping Methods in Quantitative Feedback Theory," Dyn. Sys., Meas., Control, Vol. 116, no. 2, pp. 167-177, 1994 